\title{
Optimal Control of a Fully Decentralized Quadratic Regulator
}

\author{
Laurent Lessard ${ }^{1,2}$ \\ Allerton Conference on Communication, Control, and Computing, pp. 48-54, 2012
}

\begin{abstract}
In this paper, we consider a fully decentralized control problem with two dynamically decoupled agents. The objective is to design a state-feedback controller for each agent such that a global quadratic cost is minimized. No communication, explicit or implicit, is permitted between the agents. However, the performance of the agents is coupled via the cost function as well as the process noise. We provide an explicit state-space construction of the optimal controllers, showing that the optimal controllers are dynamic, where the number of states depends on the joint covariance matrix of the process noise. The key step is a novel decomposition of the noise covariance matrix, which allows the convex program associated with the controller synthesis to be split into simpler problems and thereby solved.
\end{abstract}

\section{Introduction}

In decentralized control problems, the goal is to achieve global performance by designing local feedback policies. This happens frequently in practice. Examples include computer networks, cooperative tasks with autonomous robots, and power distribution micro-grids. The key feature of these systems is that individual agents must make decisions with only partial knowledge of the whole system's state because allowing full knowledge would be infeasible or impossible.

In this paper, we consider the simplest case of maximal decentralization; two agents cooperate to optimize a global objective, but are dynamically decoupled and unable to communicate with each other. A block diagram is shown in Figure 1, and state equations are given below.

$$
\begin{array}{ll}
\mathcal{P}_{i}: & \dot{x}_{i}=A_{i} x_{i}+B_{i} u_{i}+w_{i} \\
\mathcal{K}_{i}: & u_{i}=\gamma_{i}\left(\mathcal{X}_{i}\right)
\end{array} \quad \text { for } i=1,2
$$

where $\mathcal{X}_{i}$ is the past history of the state $x_{i}$. As depicted in Figure 1, both systems are only coupled externally; they are driven by correlated noise, and the quadratic cost function that must be optimized is coupled.

Given dynamically decoupled agents that cannot communicate, one might be tempted to think that cooperation is impossible. In other words, the two controllers

\footnotetext{
${ }^{1}$ L. Lessard is with the Department of Automatic Control at Lund University, Lund, Sweden. laurent.lessard@control.1th.se

${ }^{2}$ This work was supported by the Swedish Research Council through the LCCC Linnaeus Center
}

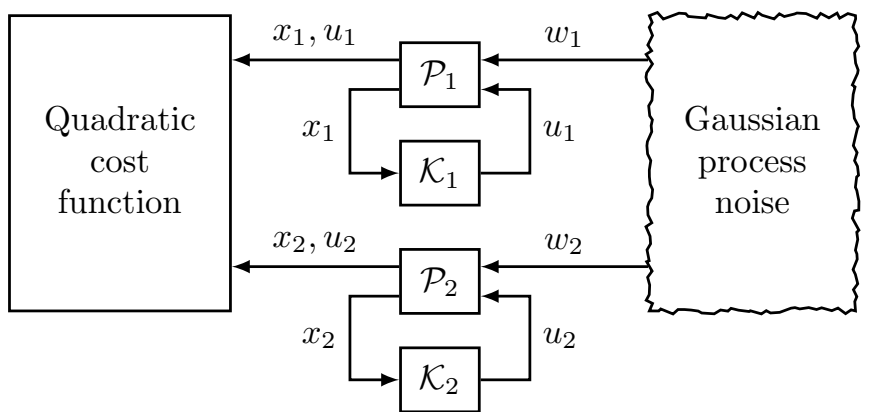

Figure 1: Block diagram showing the decentralized control architecture considered in this paper. Two feedback systems are coupled only through process noise and cost function. The goal is to jointly design state-feedback controllers $\mathcal{K}_{1}$ and $\mathcal{K}_{2}$ that minimize the expected cost.

may be designed separately without any loss of performance. There are two special cases for which this is true.

1) Coupled cost and independent noise. Despite a coupled cost, the agents have no way of estimating each other's state. The best any agent can do is design their control policy under the assumption that the other agent has zero state and input.

2) Decoupled cost and correlated noise. With a decoupled cost, actions of one agent do not alter the cost seen by the other agent. Even if the agents were to know each other's state, that information wouldn't be useful. Agents might as well assume they are alone when they design their control policy.

Roughly speaking, being able to affect all parts of the cost function isn't useful if you know nothing about the other agent's state. Similarly, knowing the other agent's state isn't useful if you have no power to affect the associated part of the cost function. In both of the cases listed above, the optimal policy for each agent is a static statefeedback controller.

In this paper, we solve the interesting case where the cost function is coupled and the noises are correlated. It turns out that in general, the optimal controller is dynamic, and its order depends on the extent to which the noises are correlated.

In the remainder of this section, we survey some relevant works from the literature and explain some of the 
notation and conventions used in the paper. In Section 2, we formally state the problem. In Section 3, we convert the problem to a useful model-matching form, which is the starting point for our solution approach. We present our main results in Sections 4 and 5, and give an illustrative example in Section 6. Finally, we end with some concluding remarks in Section 7.

\subsection{Previous Work}

The underlying controller synthesis problem considered in this paper is an example of a dynamic team decision problem. Due to the completely decentralized nature of the information structure, it is in fact a trivial example of a partially nested architecture [1]. Therefore, the optimal control policy for each agent will be linear. However, linearity alone does not guarantee that the policies will have finite memory.

It was shown more recently that a broad class of decentralized control problems, including the partially nested cases, can be convexified, and are amenable to a variety of numerical approaches $[5,6]$. When the decentralization is characterized by a sparsity constraint on the plant and controller transfer matrices, the convex program takes the form of a structured model-matching problem. for the two-agent architecture considered in this paper, the model-matching problem is given by (6)

Solutions to such problems can be explicitly constructed via vectorization [7]. However, this approach generally produces controllers with very large state dimension, and no apparent way to simplify them. One exception is the recent work of Kristalny and Shah [2], in which the authors find a minimal state-space solution for a variant of the model-matching problem considered herein (6) using a vectorization approach. An important difference with their work is that they assume a fully diagonal controller, rather than block-diagonal one. It is not clear how one would extend their approach to the case of non-SISO controllers.

State-feedback control under sparsity constraints has been well-studied, but typically under the assumption that the agents interact through dynamic coupling or explicit communication. The two-agent problem with one-way communication is solved in [9] and the $n$-agent problem with more general interconnection topologies is solved in [8]. Both of these works assume that the noise injected into each plant is independent.

The assumption of independent noise is sometimes not restrictive. For example, the general case of two agents with one-way communication and output feedback is solved in [3], and the solution is no more complicated when the noise is correlated between plants. However, the correlated noise makes a big difference when the agents cannot communicate explicitly or implicitly. As we show in this paper, such cases lead to an optimal controller whose state dimension depends on the extent to which the noise injected into both plants is correlated.

\subsection{Preliminaries}

The real and complex numbers are denoted by $\mathbb{R}$ and $\mathbb{C}$, respectively. The imaginary unit is $j$, and we denote the imaginary axis by $j \mathbb{R}$. A square matrix $A \in \mathbb{R}^{n \times n}$ is Hurwitz if all of its eigenvalues have a strictly negative real part. The set $\mathcal{L}_{2}(j \mathbb{R})$, or simply $\mathcal{L}_{2}$, is a Hilbert space of Lebesgue measurable matrix-valued functions $F: j \mathbb{R} \mapsto \mathbb{C}^{m \times n}$ with the inner product

$$
\langle F, G\rangle=\frac{1}{2 \pi} \int_{-\infty}^{\infty} \operatorname{Trace}\left[F^{*}(j \omega) G(j \omega)\right] \mathrm{d} \omega
$$

such that the inner product induced norm $\|F\|_{2}^{2}=\langle F, F\rangle$ is bounded. We will sometimes write $\mathcal{L}_{2}^{m \times n}$ to be explicit about the matrix dimensions. As is standard, $\mathcal{H}_{2}$ is a closed subspace of $\mathcal{L}_{2}$ with matrix functions analytic in the open right-half plane. $\mathcal{H}_{2}^{\perp}$ is the orthogonal complement of $\mathcal{H}_{2}$ in $\mathcal{L}_{2}$. We use the prefix $\mathcal{R}$ to indicate the subset of proper real rational functions. So $\mathcal{R} \mathcal{L}_{2}$ is the set of strictly proper rational transfer functions with no poles on the imaginary axis, and $\mathcal{R H}_{2}$ is the stable subspace of $\mathcal{R} \mathcal{L}_{2}$. It is useful to extend these sets to allow for transfer functions that are proper but not strictly proper. To this end, we have $\mathcal{R} \mathcal{L}_{2} \subset \mathcal{R} \mathcal{L}_{\infty}$ and the corresponding $\mathcal{R H}_{2} \subset \mathcal{R H}_{\infty}$. If $\mathcal{G} \in \mathcal{R} \mathcal{L}_{\infty}$, then $\mathcal{G}$ has a state-space realization

$$
\mathcal{G}=\left[\begin{array}{l|l}
A & B \\
\hline C & D
\end{array}\right]=D+C(s I-A)^{-1} B
$$

If this realization is chosen to be stabilizable and detectable, then $\mathcal{G} \in \mathcal{R} \mathcal{H}_{\infty}$ if and only if $A$ is Hurwitz, and $\mathcal{G} \in \mathcal{R H}_{2}$ if and only if $A$ is Hurwitz and $D=0$. For a thorough introduction to these topics, see [10].

A family of state-space realizations is called generically minimal if at least one member of the family is minimal. For example, consider the two families

$\mathcal{T}_{1}=\left[\begin{array}{cc|c}A_{1} & 0 & B_{1} \\ 0 & A_{2} & B_{2} \\ \hline I & I & 0\end{array}\right]$ and $\mathcal{T}_{2}=\left[\begin{array}{cc|c}A_{1} & 0 & B_{1} \\ 0 & A_{2} & 0 \\ \hline I & I & 0\end{array}\right]$

where $A_{i} \in \mathbb{R}^{n_{i} \times n_{i}}$ and $B_{i} \in \mathbb{R}^{n_{i} \times m_{i}}$ are free parameters. Then $\mathcal{T}_{1}$ is generically minimal, even though it is reducible when $A_{1}=A_{2}$. However, $\mathcal{T}_{2}$ is not generically minimal.

Many equations in this paper are expressed in terms of the solutions to Algebraic Riccati Equations (AREs). We introduce the notation $K=\operatorname{care}(A, B, C, D)$ to mean that $K=-\left(D^{\top} D\right)^{-1}\left(B^{\top} X+D^{\top} C\right)$, where $X>0$ is the stabilizing solution to the ARE

$$
\begin{aligned}
A^{\top} X+ & X A+C^{\top} C \\
& -\left(X B+C^{\top} D\right)\left(D^{\top} D\right)^{-1}\left(B^{\top} X+D^{\top} C\right)=0
\end{aligned}
$$

Whenever this notation is invoked, there will always exist a unique stabilizing solution to the associated ARE. 


\section{Problem Statement}

We now give a formal description of the problem illustrated in Figure 1. Consider the continuous-time linear time-invariant dynamical system defined by the statespace equations

$$
\begin{aligned}
{\left[\begin{array}{l}
\dot{x}_{1} \\
\dot{x}_{2}
\end{array}\right] } & =\left[\begin{array}{cc}
A_{1} & 0 \\
0 & A_{2}
\end{array}\right]\left[\begin{array}{l}
x_{1} \\
x_{2}
\end{array}\right]+\left[\begin{array}{cc}
B_{1} & 0 \\
0 & B_{2}
\end{array}\right]\left[\begin{array}{l}
u_{1} \\
u_{2}
\end{array}\right]+\left[\begin{array}{l}
N_{1} \\
N_{2}
\end{array}\right] e \\
z & =\left[\begin{array}{ll}
C_{1} & C_{2}
\end{array}\right]\left[\begin{array}{l}
x_{1} \\
x_{2}
\end{array}\right]+\left[\begin{array}{ll}
D_{1} & D_{2}
\end{array}\right]\left[\begin{array}{l}
u_{1} \\
u_{2}
\end{array}\right]
\end{aligned}
$$

where $e$ is white Gaussian noise. The noise terms entering each subsystem $w_{i}=N_{i} e$ are therefore jointly Gaussian. The states and inputs are partitioned as $x_{i}(t) \in \mathbb{R}^{n_{i}}$ and $u_{i}(t) \in \mathbb{R}^{m_{i}}$. More compactly,

$$
\begin{aligned}
& \dot{x}=A x+B u+N e \\
& z=C x+D u
\end{aligned}
$$

where $A \in \mathbb{R}^{n \times n}$ and $B \in \mathbb{R}^{n \times m}$ are block-diagonal, but $C, D$, and $N$ may be full. The two systems are coupled through the correlated noise that drives them. This fact is characterized by the joint covariance matrix

$$
\operatorname{cov}\left[\begin{array}{l}
w_{1} \\
w_{2}
\end{array}\right]=\left[\begin{array}{ll}
W_{11} & W_{12} \\
W_{21} & W_{22}
\end{array}\right]=N N^{\top}
$$

The two systems are also coupled through the infinitehorizon quadratic cost

$$
\mathcal{J}=\left(\lim _{T \rightarrow \infty} \frac{1}{T} \mathbf{E} \int_{0}^{T}\|z\|^{2} \mathrm{~d} t\right)^{1 / 2}
$$

We seek a fully decentralized linear time-invariant control policy that minimizes (4). In other words, we are interested in controllers of the form:

$$
\begin{aligned}
\mathcal{K}_{i}: & \dot{q}_{i}=A_{K i} q_{i}+B_{K i} x_{i} \\
u_{i} & =C_{K i} q_{i}+D_{K i} x_{i}
\end{aligned} \quad \text { for } i=1,2
$$

We make several assumptions regarding the matrices $A$, $B, C, D$, and $N$, and we list them below.

A1) $A$ is Hurwitz.

A2) $D$ has full column rank.

A3) $\left[\begin{array}{cc}A-j \omega I & B \\ C & D\end{array}\right]$ has full column rank for all $\omega \in \mathbb{R}$.

A4) $N$ has full row rank.

Assumption A1 is not restrictive. To see why, notice that $(A, B)$ being stabilizable is a necessary condition for the existence of a stabilizing control policy. Since $A$ and $B$ are block-diagonal, the $\mathrm{PBH}$ test implies that this condition is equivalent to $\left(A_{1}, B_{1}\right)$ and $\left(A_{2}, B_{2}\right)$ being stabilizable. Therefore, if $A_{i}$ is not Hurwitz, we can change coordinates by setting $u_{i}=F_{i} x_{i}+\tilde{u}_{i}$, where $F_{i}$ is chosen such that $A_{i}+B_{i} F_{i}$ is Hurwitz. The original and transformed subsystems for $i=1,2$ are:

$$
\begin{aligned}
\dot{x}_{i} & =A_{i} x_{i}+B_{i} u_{i} \\
\dot{q}_{i} & =A_{K i} q_{i}+B_{K i} x_{i} \\
u_{i} & =C_{K i} q_{i}+D_{K i} x_{i}
\end{aligned} \Longleftrightarrow \begin{aligned}
\dot{x}_{i} & =\left(A_{i}+B_{i} F_{i}\right) x_{i}+B_{i} \tilde{u}_{i} \\
\dot{q}_{i} & =A_{K i} q_{i}+B_{K i} x_{i} \\
\tilde{u}_{i} & =C_{K i} q_{i}+\left(D_{K i}-F_{i}\right) x_{i}
\end{aligned}
$$

So the system on the left is internally stable if and only if the one on the right is as well. Assumptions A2-A4 are standard for the centralized LQR problem, and guarantee the existence and uniqueness of an optimal controller [10].

\section{Conversion to Model-Matching Form}

The state-feedback control problem of Section 2 is a special case of a class of structured model-matching problems. Suppose $\mathcal{F}, \mathcal{G} \in \mathcal{R H}_{\infty}$, and $N$ is a real matrix. The decentralized two-player model-matching problem with one-sided dynamics is given by

$$
\begin{array}{cl}
\text { minimize } & \left\|\mathcal{F}+\mathcal{G}\left[\begin{array}{cc}
\mathcal{Q}_{1} & 0 \\
0 & \mathcal{Q}_{2}
\end{array}\right] N\right\|_{2} \\
\text { subject to } & \mathcal{Q}_{1}, \mathcal{Q}_{2} \in \mathcal{R} \mathcal{H}_{2}
\end{array}
$$

We can transform the state-feedback problem of Section 2 into a problem of type (6) via the following lemma.

Lemma 1. Suppose Assumptions A1-A4 hold for the decentralized $L Q R$ problem $(2)-(5)$. Define $\mathcal{F}$ and $\mathcal{G}$ as

$$
\mathcal{F}=\left[\begin{array}{c|c}
A & N \\
\hline C & 0
\end{array}\right] \quad \text { and } \quad \mathcal{G}=\left[\begin{array}{c|c}
A & B \\
\hline C & D
\end{array}\right]
$$

Consider the model-matching problem (6), with dimensions $\mathcal{Q}_{i} \in \mathcal{R H}_{2}^{m_{i} \times n_{i}}$. Suppose its solution is given by

$$
\mathcal{Q}_{i}=\left[\begin{array}{c|c}
\hat{A}_{i} & \hat{B}_{i} \\
\hline \hat{C}_{i} & 0
\end{array}\right] \quad \text { for } i=1,2
$$

This solution is related to the solution $u_{i}=\mathcal{K}_{i} x_{i}$ for $i=1,2$ of the original decentralized $L Q R$ problem via

$$
\mathcal{K}_{i}=\left[\begin{array}{c|c}
\hat{A}_{i}-\hat{B}_{i} B_{i} \hat{C}_{i} & \left(\hat{A}_{i}-\hat{B}_{i} B_{i} \hat{C}_{i}\right) \hat{B}_{i}-\hat{B}_{i} A_{i} \\
\hline \hat{C}_{i} & \hat{C}_{i} \hat{B}_{i}
\end{array}\right]
$$

Proof. We proceed by showing that the cost functions for both optimization problems are the same, that their domains are the same, and that $\mathcal{Q}$ defined in (8) corresponds to $\mathcal{K}$ defined in (9). The map from inputs to outputs $(e, u) \mapsto(z, x)$ is given by

$$
\left[\begin{array}{l}
z \\
x
\end{array}\right]=\left[\begin{array}{ll}
\mathcal{P}_{11} & \mathcal{P}_{12} \\
\mathcal{P}_{21} & \mathcal{P}_{22}
\end{array}\right]\left[\begin{array}{l}
e \\
u
\end{array}\right]=\left[\begin{array}{c|cc}
A & N & B \\
\hline C & 0 & D \\
I & 0 & 0
\end{array}\right]\left[\begin{array}{l}
e \\
u
\end{array}\right]
$$

where $e$ is white Gaussian noise and $w=N e$. Note that $\mathcal{P}_{11}=\mathcal{F}$ and $\mathcal{P}_{12}=\mathcal{G}$. Consider the relation

$$
\mathcal{Q}=\mathcal{K}\left(I-\mathcal{P}_{22} \mathcal{K}\right)^{-1}(s I-A)^{-1}
$$

It is straightforward to verify that (11) is satisfied by $\mathcal{Q}$ and $\mathcal{K}$ defined in (8) and (9) respectively. Substituting $u=\mathcal{K} x$, the closed-loop map $w \mapsto z$ is

$$
\begin{aligned}
\mathcal{T}_{z w} & =\mathcal{P}_{11}+\mathcal{P}_{12} \mathcal{K}\left(I-\mathcal{P}_{22} \mathcal{K}\right)^{-1} \mathcal{P}_{21} \\
& =\mathcal{F}+\mathcal{G} \mathcal{Q} N
\end{aligned}
$$


The cost function (4) is equal to the $\mathcal{H}_{2}$-norm of $\mathcal{T}_{z w}$, so we have verified that the cost functions are the same, and that $\mathcal{K}$ corresponds to $\mathcal{Q}$ via (8)-(9) and (11).

Now, we show that the domains of both optimization problems are the same. By Assumption A1, $A$ is Hurwitz. Thus, the $\mathcal{P}_{i j}$ are stable, and $\mathcal{K}$ stabilizes $\mathcal{P}$ if and only if $\mathcal{K}\left(I-\mathcal{P}_{22} \mathcal{K}\right)^{-1}$ is stable [10]. So for any stabilizing $\mathcal{K}$, if follows from (11) that $\mathcal{Q}$ is stable. Conversely, the $\mathcal{K}$ defined in (9) is stabilizing, because we can verify by direct substitution that $\mathcal{K}\left(I-\mathcal{P}_{22} \mathcal{K}\right)^{-1}$ is stable whenever $\hat{A}_{i}$ is Hurwitz. Finally, note that $\mathcal{P}_{22}$ is block-diagonal, so $\mathcal{Q}$ is block-diagonal whenever $\mathcal{K}$ is block-diagonal.

Note that Lemma 1 produces a realization for $\mathcal{K}$ in $(9)$ that has the same order as the realization for $\mathcal{Q}$ in (8), but it does not guarantee that the realization for $\mathcal{K}$ will be minimal if we started with a minimal realization for $\mathcal{Q}$. Indeed, the two special cases listed in Section 1 have optimal controllers that are static, yet the corresponding $\mathcal{Q}_{i}$ are dynamic.

\section{Model-Matching Solution}

In this section, we present the solution to the modelmatching problem given in (6). We suppose $\mathcal{F}, \mathcal{G} \in \mathcal{R} \mathcal{H}_{\infty}$ have a stabilizable and detectable joint realization

$$
\left[\begin{array}{ll}
\mathcal{F} & \mathcal{G}
\end{array}\right]=\left[\begin{array}{c|cc}
A & \Gamma & B \\
\hline C & 0 & D
\end{array}\right]
$$

When the model-matching problem comes from the decentralized LQR problem (2)-(5) as in Lemma 1, $A$ and $B$ are block-diagonal and $\Gamma=N$. Our approach does not rely on these assumptions, so we will drop them in favor of solving the more general model-matching problem for which $A$ and $B$ have no structure, and $\Gamma$ is arbitrary. In addition to Assumptions A1-A4, we also assume that

$$
N N^{\top}=W=\left[\begin{array}{cc}
I & Y \\
Y^{\top} & I
\end{array}\right]
$$

There is no loss of generality in this assumption, because $W>0$ by Assumption A4, and we can scale $\mathcal{Q}_{1}$ and $\mathcal{Q}_{2}$ by $W_{11}^{1 / 2}$ and $W_{22}^{1 / 2}$ respectively. The key step in solving the model-matching problem is a decomposition of $W$, which begins with the full singular value decomposition

$$
Y=\left[\begin{array}{ll}
U_{1} & \bar{U}_{1}
\end{array}\right]\left[\begin{array}{cc}
\Sigma & 0 \\
0 & 0
\end{array}\right]\left[\begin{array}{ll}
U_{2} & \bar{U}_{2}
\end{array}\right]^{\top}
$$

where $\left[\begin{array}{ll}U_{i} & \bar{U}_{i}\end{array}\right]$ is orthogonal, $\Sigma$ is a diagonal matrix with entries $\sigma_{1}, \ldots, \sigma_{r}$, and $r=\operatorname{rank}(Y)$. Note that because $W>0$, we have $1>\sigma_{1} \geq \cdots \geq \sigma_{r}>0$.

We also require some new definitions. First, define $e_{k} \in \mathbb{R}^{r \times 1}$ for $k=1, \ldots, r$ to be the $k^{\text {th }}$ unit vector, with 1 in the $k^{\text {th }}$ position and 0 everywhere else. Similarly, define block versions $E_{1}=\left[\begin{array}{ll}I & 0\end{array}\right]^{\top}$ and $E_{2}=\left[\begin{array}{ll}0 & I\end{array}\right]^{\top}$, where the sizes of the blocks are inferred by context. Finally, define

$$
\begin{aligned}
\tilde{A} & =\left[\begin{array}{ll}
A & \\
& A
\end{array}\right], \tilde{B}=\left[\begin{array}{l}
B E_{1} E_{1}^{\top} \\
B E_{2} E_{2}^{\top}
\end{array}\right], \beta_{k}=\sqrt{\frac{\sigma_{k}}{1-\sigma_{k}}}, \\
\tilde{C}_{k} & =\left[\begin{array}{cc}
\beta_{k} C & \beta_{k} C \\
C & 0 \\
0 & C
\end{array}\right], \tilde{D}_{k}=\left[\begin{array}{c}
\beta_{k} D \\
D E_{1} E_{1}^{\top} \\
D E_{2} E_{2}^{\top}
\end{array}\right] \\
\tilde{N}_{k} & =\left[\begin{array}{cc}
I & \sigma_{k} I \\
\sigma_{k} I & I
\end{array}\right]^{-1}\left[\begin{array}{l}
\Gamma N^{\top} E_{1} U_{1} \\
\Gamma N^{\top} E_{2} U_{2}
\end{array}\right] \\
K_{i} & =\operatorname{care}\left(A, B E_{i}, C, D E_{i}\right) \\
\tilde{K}_{k} & =\operatorname{care}\left(\tilde{A}, \tilde{B}, \tilde{C}_{k}, \tilde{D}_{k}\right)
\end{aligned}
$$

where $i=1,2$ and $k=1, \ldots, r$. The main result of this section is given below, and a complete proof is provided in Section 8.

Theorem 2. Suppose $\mathcal{F}, \mathcal{G} \in \mathcal{R} \mathcal{H}_{\infty}$ have a stabilizable and detectable joint realization given by (12), $N$ is a real matrix of the form (13), and Assumptions A1-A4 hold. The solution to

$$
\begin{array}{ll}
\text { minimize } & \left\|\mathcal{F}+\mathcal{G}\left[\begin{array}{cc}
\mathcal{Q}_{1} & 0 \\
0 & \mathcal{Q}_{2}
\end{array}\right] N\right\|_{2} \\
\text { subject to } & \mathcal{Q}_{1}, \mathcal{Q}_{2} \in \mathcal{R H}_{2}
\end{array}
$$

is given by

$$
\mathcal{Q}_{i}=\left[\begin{array}{cccc|c}
\tilde{A}+\tilde{B} \tilde{K}_{1} & & & & \tilde{N}_{1} e_{1} e_{1}^{\top} U_{i}^{\top} \\
& \ddots & & & \vdots \\
& & & & \\
& & & & \\
& & & A+B \tilde{K}_{r} & \\
\tilde{N}_{r} e_{r} e_{r}^{\top} U_{i}^{\top} \\
\Gamma N^{\top} E_{i} \bar{U}_{i} \bar{U}_{i}^{\top} \\
\hline E_{i}^{\top} \tilde{K}_{1} & \cdots & E_{i}^{\top} \tilde{K}_{r} & K_{i} & 0
\end{array}\right]
$$

where the relevant quantities are defined in (14)-(15). The realization for $\mathcal{Q}_{i}$ given above is generically minimal.

The formula for $\mathcal{Q}_{i}$ in Theorem 2 becomes simpler for boundary values of $r$. Since $r=\operatorname{rank}(Y)$ and $Y \in \mathbb{R}^{n_{1} \times n_{2}}$, we have $0 \leq r \leq \min \left(n_{1}, n_{2}\right)$. When $r=0$, the $\tilde{A}+\tilde{B} \tilde{K}_{k}$ terms vanish. Similarly, when $r=n_{i}$, the $A+B E_{i} K_{i}$ term vanishes. In general,

$$
\text { \# of states in } \mathcal{Q}_{i}= \begin{cases}2 n r+n & \text { if } 0 \leq r<\min \left(n_{1}, n_{2}\right) \\ 2 n r & \text { if } r=n_{i}\end{cases}
$$

\section{State-Feedback Solution}

In this section, we combine Theorem 2 and Lemma 1 to give a complete solution to the state-feedback problem of Section 2. Upon reintroduction of the constraints that $\Gamma=N$ and that $A$ and $B$ are block-diagonal, the solution in Theorem 2 simplifies considerably. Recalling the 
partitions of the matrices introduced in (2), define

$$
\begin{aligned}
& \check{C}_{k}=\left[\begin{array}{c}
\beta_{k} C \\
C_{1} E_{1}^{\top} \\
C_{2} E_{2}^{\top}
\end{array}\right] \quad \check{D}_{k}=\left[\begin{array}{c}
\beta_{k} D \\
D_{1} E_{1}^{\top} \\
D_{2} E_{2}^{\top}
\end{array}\right] \quad \check{N}=\left[\begin{array}{c}
U_{1} \\
U_{2}
\end{array}\right] \\
& \hat{K}_{i}=\operatorname{care}\left(A_{i}, B_{i}, C_{i}, D_{i}\right) \\
& \check{K}_{k}=\operatorname{care}\left(A, B, \check{C}_{k}, \check{D}_{k}\right) \\
& A_{L}=\left[\begin{array}{c}
A+B \check{K}_{1} \\
\ddots \\
A+B \check{K}_{r}
\end{array}\right] B_{L i}=\left[\begin{array}{c}
\check{N} e_{1} e_{1}^{\top} U_{i}^{\top} \\
\vdots \\
\check{N} e_{r} e_{r}^{\top} U_{i}^{\top}
\end{array}\right] \\
& C_{K i}=\left[\begin{array}{lll}
E_{i}^{\top} \check{K}_{1}-\hat{K}_{i} E_{i}^{\top} & \cdots & E_{i}^{\top} \check{K}_{r}-\hat{K}_{i} E_{i}^{\top}
\end{array}\right] \\
& D_{K i}=\hat{K}_{i} \bar{U}_{i} \bar{U}_{i}^{\top}+E_{i}^{\top} \sum_{k=1}^{r} \check{K}_{k} \check{N} e_{k} e_{k}^{\top} U_{i}^{\top}
\end{aligned}
$$

where $i=1,2$ and $k=1, \ldots, r$. Recall that $\beta_{k}$ was previously defined in (15). The main result of this section is below, and a complete proof is provided in Section 8 .

Theorem 3. Suppose Assumptions A1-A4 hold for the decentralized LQR problem (2)-(5). Further suppose that $N$ satisfies (13)-(14). The optimal decentralized LQR controller $u_{i}=\mathcal{K}_{i} x_{i}$ is given by

$\mathcal{K}_{i}=\left[\begin{array}{c|c}A_{L}-B_{L i} B_{i} C_{K i} & A_{L} B_{L i}-B_{L i}\left(A_{i}+B_{i} D_{K i}\right) \\ \hline C_{K i} & D_{K i}\end{array}\right]$

where the relevant quantities are defined in (16). The realization for $\mathcal{K}_{i}$ given above is generically minimal if $r<\min \left(n_{1}, n_{2}\right)$.

For the case where $r=n_{i}$, an additional reduction can be made. See Section 8 for details. In general,

$$
\text { \# of states in } \mathcal{K}_{i}= \begin{cases}n r & \text { if } 0 \leq r<\min \left(n_{1}, n_{2}\right) \\ n r-n_{i} & \text { if } r=n_{i}\end{cases}
$$

As mentioned in Section 1, the case of uncorrelated noise leads to a static optimal controller. To verify this, note that uncorrelated noise implies that $Y=0$, so $r=0$. In this case, $\bar{U}_{i}$ is square and orthogonal, so $\bar{U}_{i} \bar{U}_{i}^{\top}=I$. It follows that the optimal controller is $\mathcal{K}_{i}=D_{K i}=$ $\hat{K}_{i}$. This is precisely the static state-feedback policy each agent would employ if they were ignoring the other agent.

\section{Example}

In this section, we illustrate the results of this paper by solving a simple decentralized LQR problem. Suppose two ships move on parallel trajectories with velocities $v_{1}$ and $v_{2}$ respectively, subject to the dynamics

$$
\begin{aligned}
& \dot{v}_{1}=-2 v_{1}+u_{1}+w_{1} \\
& \dot{v}_{2}=-3 v_{2}+u_{2}+w_{2}
\end{aligned}
$$

The Gaussian disturbances $w_{i}$ have unit variance, and are correlated as $\mathbf{E}\left(w_{1} w_{2}\right)=0.8$. Each ship knows its own velocity $v_{i}$, and must control its thruster input $u_{i}$. The objective is for the ships to match their velocities while keeping thruster effort low. We use the cost function

$$
\mathcal{J}=\left(\lim _{T \rightarrow \infty} \frac{1}{T} \mathbf{E} \int_{0}^{T}\left[\left(v_{1}-v_{2}\right)^{2}+0.01\left(u_{1}^{2}+u_{2}^{2}\right)\right] \mathrm{d} t\right)^{1 / 2}
$$

The following table compares various policies.

\begin{tabular}{lll} 
Policy & Control Law & Cost \\
\hline No control & $u_{1}=u_{2}=0$ & 0.3109 \\
& $u_{1}=-8.1980 v_{1}$ & 0.2808 \\
Selfish & $u_{2}=-7.4403 v_{2}$ & \\
& $\dot{q}_{1}=-13.79 q_{1}-0.2659 v_{1}$ & \\
Optimal & $u_{1}=4.255 q_{1}-3.013 v_{1}$ & 0.2300 \\
& $\dot{q}_{2}=-13.52 q_{2}+0.2659 v_{2}$ & \\
& $u_{2}=4.255 q_{2}-2.279 v_{2}$ & \\
Centralized & $u_{1}=-6.3095 v_{1}+5.9121 v_{2}$ & 0.1567 \\
& $u_{2}=5.9121 v_{1}-5.6051 v_{2}$ & \\
\hline
\end{tabular}

The selfish policy assumes the ships ignore each other. This amounts to solving two centralized LQR problems, and the solution is $u_{i}=\hat{K}_{i}$. The optimal decentralized policy was found using Theorem 3. As expected, each ship's controller is dynamic with a state dimension of 1 because $r=n_{1}=n_{2}=1$. Finally, the centralized policy allows the ships to know each other's velocities. It is given by $u=K v$, where $K=\operatorname{care}(A, B, C, D)$. As expected, the centralized policy is the best one, and the optimal decentralized policy outperforms the selfish one.

\section{Concluding Remarks}

We presented an explicit state-space solution to a class of fully decentralized state-feedback problems with two agents. When the cost is coupled between both agents, the optimal controller is dynamic and has a state dimension that depends on the extent to which the noises affecting the agents are correlated.

It is not clear how one might extend the solution presented herein to problems involving more than two agents, or to output feedback architectures where agents only have access to noisy measurements of their own states. These are possible avenues for future research.

\section{Proofs of the Main Results}

Proof of Theorem 2. It is convenient to introduce frequency-domain versions of Assumptions A1-A4.

B1) $\mathcal{F}$ is strictly proper.

B2) $\mathcal{G}(j \omega)$ has full column-rank for all $\omega \in \mathbb{R}$, including $\omega=\infty$.

B3) $N$ has full row-rank. 
If the joint realization for $\left[\begin{array}{ll}\mathcal{F} & \mathcal{G}\end{array}\right]$ in $(12)$ is chosen to be a stabilizable and detectable, Assumptions A1-A4 are equivalent to Assumptions B1-B3. Again, these assumptions are made to guarantee the existence and uniqueness of a solution to (6).

The model-matching problem (6) has an optimality condition, which follows from embedding the problem into the Hilbert space $\mathcal{L}_{2}$ and using the projection theorem [4]. We state the result as a proposition.

Proposition 4. Suppose $\mathcal{F}, \mathcal{G} \in \mathcal{R H}_{\infty}, N$ is a real matrix, and Assumptions B1-B3 are satisfied. Then $\mathcal{Q}_{1}, \mathcal{Q}_{2}$ are optimal for (6) if and only if

$$
\mathcal{G}^{*} \mathcal{F} N^{\top}+\mathcal{G}^{*} \mathcal{G}\left[\begin{array}{cc}
\mathcal{Q}_{1} & 0 \\
0 & \mathcal{Q}_{2}
\end{array}\right] N N^{\top} \in\left[\begin{array}{cc}
\mathcal{H}_{2}^{\perp} & \mathcal{L}_{2} \\
\mathcal{L}_{2} & \mathcal{H}_{2}^{\perp}
\end{array}\right]
$$

Pick out the 11 and 22 blocks of (17), and substitute the decomposition of $N$ from (13)-(14).

$$
\begin{gathered}
{\left[\mathcal{G}^{*} \mathcal{F} N^{\top}\right]_{11}+E_{1}^{\top} \mathcal{G}^{*} \mathcal{G}\left[\begin{array}{c}
\mathcal{Q}_{1} \\
\mathcal{Q}_{2} U_{2} \Sigma U_{1}^{\top}
\end{array}\right] \in \mathcal{H}_{2}^{\perp}} \\
{\left[\mathcal{G}^{*} \mathcal{F} N^{\top}\right]_{22}+E_{2}^{\top} \mathcal{G}^{*} \mathcal{G}\left[\begin{array}{c}
\mathcal{Q}_{1} U_{1} \Sigma U_{2}^{\top} \\
\mathcal{Q}_{2}
\end{array}\right] \in \mathcal{H}_{2}^{\perp}}
\end{gathered}
$$

Since $\bar{U}_{i}$ is an orthogonal completion of $U_{i}$, i.e. $\left[\begin{array}{ll}U_{i} & \bar{U}_{i}\end{array}\right]$ is orthogonal, we may write

$$
\mathcal{Q}_{i}=\left(\mathcal{Q}_{i} U_{i}\right) U_{i}^{\top}+\left(\mathcal{Q}_{i} \bar{U}_{i}\right) \bar{U}_{i}^{\top} \quad \text { for } i=1,2
$$

Rather than solving for $\mathcal{Q}_{i}$, we will solve for the projections $\mathcal{Q}_{i} U_{i}$ and $\mathcal{Q}_{i} \bar{U}_{i}$, and reconstruct $\mathcal{Q}_{i}$ via (20). Multiplication of (18)-(19) by the $\bar{U}_{i}$ yields the following.

$$
\begin{aligned}
& {\left[\mathcal{G}^{*} \mathcal{F} N^{\top}\right]_{11} \bar{U}_{1}+\left[\mathcal{G}^{*} \mathcal{G}\right]_{11}\left(\mathcal{Q}_{1} \bar{U}_{1}\right) \in \mathcal{H}_{2}^{\perp}} \\
& {\left[\mathcal{G}^{*} \mathcal{F} N^{\top}\right]_{22} \bar{U}_{2}+\left[\mathcal{G}^{*} \mathcal{G}\right]_{22}\left(\mathcal{Q}_{2} \bar{U}_{2}\right) \in \mathcal{H}_{2}^{\perp}}
\end{aligned}
$$

Multiplication of (18)-(19) by the $U_{i}$ yields the following.

$$
\begin{aligned}
& {\left[\mathcal{G}^{*} \mathcal{F} N^{\top}\right]_{11} U_{1}+E_{1}^{\top} \mathcal{G}^{*} \mathcal{G}\left[\begin{array}{c}
\mathcal{Q}_{1} U_{1} \\
\mathcal{Q}_{2} U_{2} \Sigma
\end{array}\right] \in \mathcal{H}_{2}^{\perp}} \\
& {\left[\mathcal{G}^{*} \mathcal{F} N^{\top}\right]_{22} U_{2}+E_{2}^{\top} \mathcal{G}^{*} \mathcal{G}\left[\begin{array}{c}
\mathcal{Q}_{1} U_{1} \Sigma \\
\mathcal{Q}_{2} U_{2}
\end{array}\right] \in \mathcal{H}_{2}^{\perp}}
\end{aligned}
$$

Taking advantage of the diagonal form of $\Sigma$, we consider (23)-(24) one column at a time. For $k=1, \ldots, r$,

$$
\begin{aligned}
& {\left[\mathcal{G}^{*} \mathcal{F} N^{\top}\right]_{11} U_{1} e_{k}+E_{1}^{\top} \mathcal{G}^{*} \mathcal{G}\left[\begin{array}{r}
\mathcal{Q}_{1} U_{1} e_{k} \\
\sigma_{k} \mathcal{Q}_{2} U_{2} e_{k}
\end{array}\right] \in \mathcal{H}_{2}^{\perp}} \\
& {\left[\mathcal{G}^{*} \mathcal{F} N^{\top}\right]_{22} U_{2} e_{k}+E_{2}^{\top} \mathcal{G}^{*} \mathcal{G}\left[\begin{array}{r}
\sigma_{k} \mathcal{Q}_{1} U_{1} e_{k} \\
\mathcal{Q}_{2} U_{2} e_{k}
\end{array}\right] \in \mathcal{H}_{2}^{\perp}}
\end{aligned}
$$

Now combine (25)-(26) into a single equation

$$
\begin{aligned}
& {\left[\begin{array}{l}
{\left[\mathcal{G}^{*} \mathcal{F} N^{\top}\right]_{11} U_{1}} \\
{\left[\mathcal{G}^{*} \mathcal{F} N^{\top}\right]_{22} U_{2}}
\end{array}\right] e_{k}} \\
& \quad+\left[\begin{array}{rr}
{\left[\mathcal{G}^{*} \mathcal{G}\right]_{11}} & \sigma_{k}\left[\mathcal{G}^{*} \mathcal{G}\right]_{12} \\
\sigma_{k}\left[\mathcal{G}^{*} \mathcal{G}\right]_{21} & {\left[\mathcal{G}^{*} \mathcal{G}\right]_{22}}
\end{array}\right]\left[\begin{array}{l}
\mathcal{Q}_{1} U_{1} e_{k} \\
\mathcal{Q}_{2} U_{2} e_{k}
\end{array}\right] \in \mathcal{H}_{2}^{\perp}
\end{aligned}
$$

Each of the optimality conditions (21)-(22) and (27) for $k=1, \ldots, r$ correspond to independent centralized model-matching problem. Namely, (21) corresponds to

$$
\begin{array}{cl}
\text { minimize } & \left\|\mathcal{F} N^{\top} E_{1} \bar{U}_{1}+\mathcal{G} E_{1}\left(\mathcal{Q}_{1} \bar{U}_{1}\right)\right\|_{2} \\
\text { subject to } & \left(\mathcal{Q}_{1} \bar{U}_{1}\right) \in \mathcal{R H}_{2}
\end{array}
$$

and (22) corresponds to

$$
\begin{array}{cl}
\text { minimize } & \left\|\mathcal{F} N^{\top} E_{2} \bar{U}_{2}+\mathcal{G} E_{2}\left(\mathcal{Q}_{2} \bar{U}_{2}\right)\right\|_{2} \\
\text { subject to } & \left(\mathcal{Q}_{2} \bar{U}_{2}\right) \in \mathcal{R} \mathcal{H}_{2}
\end{array}
$$

Finally, for $k=1, \ldots, r$, Equation (27) corresponds to

$$
\begin{array}{ll}
\min & \left\|\left[\begin{array}{c}
0 \\
\mathcal{F} N^{\top} E_{1} U_{1} e_{k} \\
\mathcal{F} N^{\top} E_{2} U_{2} e_{k}
\end{array}\right]+\left[\begin{array}{c}
\sqrt{\sigma_{k}\left(1-\sigma_{k}\right)} \mathcal{G} \\
\left(1-\sigma_{k}\right) \mathcal{G} E_{1} E_{1}^{\top} \\
\left(1-\sigma_{k}\right) \mathcal{G} E_{2} E_{2}^{\top}
\end{array}\right]\left[\begin{array}{c}
\mathcal{Q}_{1} U_{1} e_{k} \\
\mathcal{Q}_{2} U_{2} e_{k}
\end{array}\right]\right\|_{2} \\
\text { s.t. } & {\left[\begin{array}{l}
\mathcal{Q}_{1} U_{1} e_{k} \\
\mathcal{Q}_{2} U_{2} e_{k}
\end{array}\right] \in \mathcal{R} \mathcal{H}_{2}}
\end{array}
$$

The optimization problems (28)-(30) are centralized, meaning that unlike (6), there are no sparsity constraints on the optimization variables. The solution to such problems is well-known, and stated below as a proposition.

Proposition 5. Suppose $\mathcal{F}, \mathcal{G} \in \mathcal{R H}_{\infty}$ satisfy Assumptions B1-B2, and have a joint stabilizable and detectable realization given by (12). The model-matching problem

$$
\begin{array}{ll}
\text { minimize } & \|\mathcal{F}+\mathcal{G} \mathcal{Q}\|_{2} \\
\text { subject to } & \mathcal{Q} \in \mathcal{R} \mathcal{H}_{2}
\end{array}
$$

has a unique solution, given by

$$
\mathcal{Q}_{o p t}=\left[\begin{array}{c|c}
A+B K & \Gamma \\
\hline K & 0
\end{array}\right]
$$

where $K=\operatorname{care}(A, B, C, D)$.

We now have all the ingredients required to solve the decentralized model-matching problem with one-sided dynamics (6). First, apply Proposition 5 to (28)-(29). We find that the optimal $\mathcal{Q} \bar{U}_{1}$ and $\mathcal{Q} \bar{U}_{2}$ are given by

$$
\mathcal{Q}_{i} \bar{U}_{i}=\left[\begin{array}{c|c}
A+B E_{i} K_{i} & \Gamma N^{\top} E_{i} \bar{U}_{i} \\
\hline K_{i} & 0
\end{array}\right] \quad \text { for } i=1,2
$$

where $K_{i}=\operatorname{care}\left(A, B E_{i}, C, D E_{i}\right)$. Next, apply Proposition 5 to $(30)$. One can show after some algebraic manipulation that the $k^{\text {th }}$ column of the optimal $\mathcal{Q} U_{1}$ and $\mathcal{Q} U_{2}$ is given by

$$
\left[\begin{array}{c}
\mathcal{Q}_{1} U_{1} e_{k} \\
\mathcal{Q}_{2} U_{2} e_{k}
\end{array}\right]=\left[\begin{array}{c|c}
\tilde{A}+\tilde{B} \tilde{K}_{k} & \tilde{N}_{k} e_{k} \\
\hline \tilde{K}_{k} & 0
\end{array}\right] \text { for } k=1, \ldots, r
$$

where $\tilde{K}_{k}=\operatorname{care}\left(\tilde{A}, \tilde{B}, \tilde{C}_{k}, \tilde{D}_{k}\right)$, and the new quantities are defined in (15). Finally, we obtain the optimal $\mathcal{Q}_{1}$ and $\mathcal{Q}_{2}$ by substituting (32) and (33) into (20) and assembling the augmented state-space realizations.

One can verify that the realizations obtained for $\mathcal{Q}_{1}$ and $\mathcal{Q}_{2}$ are generically minimal by testing problem instances generated from random data. 
Proof of Theorem 3. First, consider the optimal $\mathcal{Q}_{i} \bar{U}_{i}$ from (32). Now impose the constraint that $A$ and $B$ are block-diagonal of the form (2), and that $\Gamma=N$. Making use of the identities (13)-(14), we can simplify the expression to

$$
\mathcal{Q}_{i} \bar{U}_{i}=\left[\begin{array}{c|c}
A_{i}+B_{i} \hat{K}_{i} & \bar{U}_{i} \\
\hline \hat{K}_{i} & 0
\end{array}\right] \quad \text { for } i=1,2
$$

where $\hat{K}_{i}=\operatorname{care}\left(A_{i}, B_{i}, C_{i}, D_{i}\right)$. Now consider the optimal $\mathcal{Q}_{i} U_{i}$ from (33). Similar algebraic manipulations reveal the simpler formula

$$
\left[\begin{array}{c}
\mathcal{Q}_{1} U_{1} e_{k} \\
\mathcal{Q}_{2} U_{2} e_{k}
\end{array}\right]=\left[\begin{array}{c|c}
A+B \check{K}_{k} & \check{N} e_{k} \\
\hline \check{K}_{k} & 0
\end{array}\right] \text { for } k=1, \ldots, r
$$

where $\check{K}_{k}=\operatorname{care}\left(A, B, \check{C}_{k}, \check{D}_{k}\right)$. Note that in (34)-(35), not only does the number of states decrease when compared to (32)-(33), but the size of the associated AREs shrinks as well. In the case where $r<\min \left(n_{1}, n_{2}\right)$, the expression for $\mathcal{Q}_{i}$ from Theorem 2 simplifies to

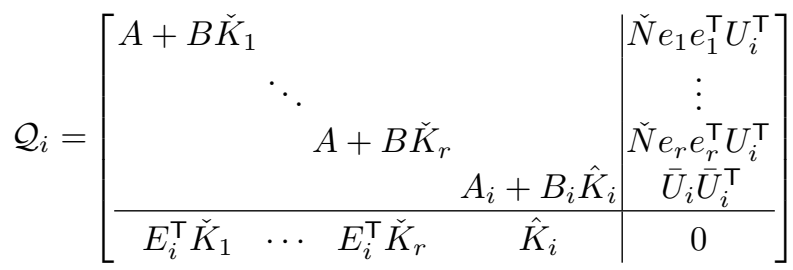

If $r=n_{i}$, then the last block of states in $\mathcal{Q}_{i}$, which are associated with $A_{i}+B_{i} \hat{K}_{i}$, vanish. We may now apply Lemma 1 to find a state-space realization of the solution to the state-feedback problem. It turns out that if we directly apply Lemma 1 , the resulting $\mathcal{K}_{i}$ is not generically minimal. This fact is made evident by transforming $\mathcal{K}_{i}$ via $\left(A_{K i}, B_{K i}, C_{K i}, D_{K i}\right) \mapsto$ $\left(T A_{K i} T^{-1}, T B_{K i}, C_{K i} T^{-1}, D_{K i}\right)$, where

$$
T=\left[\begin{array}{cccc}
I & \cdots & 0 & 0 \\
\vdots & \ddots & \vdots & \vdots \\
0 & \cdots & I & 0 \\
E_{i}^{\top} & \cdots & E_{i}^{\top} & I
\end{array}\right]
$$

In these new coordinates, the last block of states, which has size $n_{i}$, is not controllable and may be removed.

One can verify that the final realizations obtained for $\mathcal{K}_{1}$ and $\mathcal{K}_{2}$ are generically minimal when $r<\min \left(n_{1}, n_{2}\right)$ by testing problem instances generated from random data. For the extreme case where $r=n_{1}$, an additional reduction can be made. A straightforward computation shows that under the transformation

$$
\bar{T}=\left[\begin{array}{cccc}
I & I & \cdots & I \\
0 & I & \cdots & 0 \\
\vdots & \vdots & \ddots & \vdots \\
0 & 0 & \cdots & I
\end{array}\right]
$$

the first $n_{1}$ states are not controllable and may be removed. Therefore, the state dimension of the optimal $\mathcal{K}_{1}$ is reduced by $n_{1}$. Similarly, if $r=n_{2}$, the state dimension of $\mathcal{K}_{2}$ is further reduced by $n_{2}$.

\section{References}

[1] Y-C. Ho and K-C. Chu. Team decision theory and information structures in optimal control problems-Part I. IEEE Transactions on Automatic Control, 17(1):15-22, 1972.

[2] Maxim Kristalny and Parikshit Shah. On the fully decentralized two-block $\mathcal{H}_{2}$ model matching with one-sided dynamics. In American Control Conference, 2012. to appear.

[3] L. Lessard and S. Lall. A state-space solution to the two-player decentralized optimal control problem. In Allerton Conference on Communication, Control, and Computing, pages 1599-1564, 2011.

[4] D.G. Luenberger. Optimization by vector space methods. Wiley-Interscience, 1997.

[5] Xin Qi, M.V. Salapaka, P.G. Voulgaris, and M. Khammash. Structured optimal and robust control with multiple criteria: a convex solution. IEEE Transactions on Automatic Control, 49(10):16231640, 2004.

[6] M. Rotkowitz and S. Lall. A characterization of convex problems in decentralized control. IEEE Transactions on Automatic Control, 51(2):274-286, 2006.

[7] M. Rotkowitz and S. Lall. Convexification of optimal decentralized control without a stabilizing controller. In Proceedings of the International Symposium on Mathematical Theory of Networks and Systems (MTNS), pages 1496-1499, 2006.

[8] P. Shah and P. A. Parrilo. $\mathcal{H}_{2}$-optimal decentralized control over posets: A state space solution for state-feedback. In IEEE Conference on Decision and Control, pages 6722-6727, 2010.

[9] J. Swigart and S. Lall. An explicit state-space solution for a decentralized two-player optimal linearquadratic regulator. In American Control Conference, pages 6385-6390, 2010.

[10] K. Zhou, J. Doyle, and K. Glover. Robust and optimal control. Prentice-Hall, 1995. 